\title{
Isolamento de leveduras patogênicas da microbiota superficial de tamanduás mantidos em cativeiro
}

\author{
Isolation of pathogenic yeasts from the surface microbiota of anteaters held in captivity \\ Aislamiento de levaduras patogénicas de la microbiota superficial de osos hormigueros en \\ cautividad
}

Recebido: 06/12/2021 | Revisado: 11/12/2021 | Aceito: 12/12/2021 | Publicado: 21/12/2021

Henri Donnarumma Levy Bentubo

ORCID: https://orcid.org/0000-0003-0091-2504 Universidade Paulista, Brasil Universidade do Vale do Paraíba, Brasil

E-mail: hbentubo@yahoo.com.br

Flávia Regina Miranda

ORCID: https://orcid.org/0000-0002-3804-3781 Grupo de trabalho pela conservação do tamanduá no Brasil, Brasil

Universidade Estadual de Santa Cruz, Brasil E-mail: flaviamiranda@yahoo.com

Cátia Dejuste de Paula

ORCID: https://orcid.org/0000-0001-9769-4915 Universidade Federal Fluminense, Brasil E-mail: cdejuste@gmail.com

Selene Dall'Acqua Coutinho ORCID: https://orcid.org/0000-0001-9715-6500 Universidade Paulista, Brasil E-mail: selene@uol.com.br

\begin{abstract}
Resumo
A composição da microbiota fúngica do pelame de animais silvestres ainda é pouco conhecida. Estabelecer parâmetros microbiológicos que permitam prever eventos infecciosos oportunistas nesses animais pode ser útil na preservação de espécies amaçadas de extinção. O objetivo dessa investigação foi isolar e identificar leveduras de potencial patogênico do pelame de tamanduás mantidos em cativeiro. Vinte e sete tamanduás, provenientes da Fundação Parque Zoológico de São Paulo (FPZSP) e Parque Municipal Quinzinho de Barros (Zôo-Sorocaba, SP) foram pesquisados. Catorze espécimes serão de tamanduá-bandeira (Mymercophaga tridactyla) e 13 de tamanduámirim (Tamandua tetradactyla), dos quais, 63\% machos e 37\%, fêmeas. A técnica do quadrado do carpete foi empregada na obtenção das amostras de pelame. As leveduras isoladas foram identificadas por meio de suas características morfológicas e por método semi-automatizado ID-32 ${ }^{\circledR}$. Para descrever as variáveis obtidas por meio do instrumento de pesquisa, foram verificadas a frequência de ocorrência e os resultados foram expressos em valores relativos. Foram isoladas, no total, 33 leveduras a partir das amostras de pelame dos 27 tamanduás. As espécies de leveduras isoladas foram: oito Candida guilliermondii (24,2\%), três $C$. famata $(9,1 \%)$, três $C$. kefyr $(9,1 \%)$, duas $C$. glabrata (6,1\%), três Cryptococcus laurentii (9,1\%), um C. humicola (3,0\%), seis Geotrichum candidum (18,2\%), três Malassezia pachydermatis (9.1\%), duas Rhodotorula glutinis $(6,1 \%)$ e dois Trichosporon asahii (6,1\%). Pode-se concluir que leveduras reconhecidamente patogênicas podem colonizar a microbiota do tegumento de tamanduásbandeira e tamanduás-mirim mantidos em cativeiro e representam potencial risco de infecção oportunista para esses animais.
\end{abstract}

Palavras-chave: Leveduras patogênicas; Microbiota normal; Tamanduá-bandeira; Tamanduá-mirim.

\begin{abstract}
The composition of the fungal microbiota in the fur of wild animals is still poorly known. Establishing microbiological parameters that allow predicting opportunistic infectious events in these animals can be useful in the preservation of endangered species. The aim of this investigation was to isolate and identify potentially pathogenic yeasts from the coat of anteaters kept in captivity. Twenty-seven anteaters, from the Parque Zoológico de São Paulo Foundation (FPZSP) and Quinzinho de Barros Municipal Park (Zôo-Sorocaba, SP) were surveyed. Fourteen specimens will be giant anteater (Mymercophaga tridactyla) and 13 anteater (Tamandua tetradactyla), of which 63\% males and $37 \%$ females. The carpet square technique was used to obtain the skin samples. Isolated yeasts were identified by their morphological characteristics and by semi-automated ID-32C $\mathrm{C}^{\mathrm{TM}}$ method. To describe the variables obtained through the research instrument, the frequency of occurrence was verified and the results were expressed in relative values. A total of 33 yeasts were isolated from the skin samples of the 27 anteaters. The yeast species isolated
\end{abstract}


were: eight Candida guilliermondii (24.2\%), three C. famata (9.1\%), three C. kefyr (9.1\%), two C. glabrata (6.1\%), three Cryptococcus laurentii (9.1\%), one C. humicola (3.0\%), six Geotrichum candidum (18.2\%), three Malassezia pachydermatis $(9.1 \%)$, two Rhodotorula glutinis $(6.1 \%)$ and two Trichosporon asahii $(6.1 \%)$. It can be concluded that yeasts known to be pathogenic can colonize the integument microbiota of giant anteaters and anteaters kept in captivity and represent a potential risk of opportunistic infection for these animals.

Keywords: Pathogenic yeasts; Normal microbiota; Giant anteater; Baby anteater.

\section{Resumen}

La composición de la microbiota fúngica en el pelaje de los animales salvajes es aún poco conocida. Establecer parámetros microbiológicos que permitan predecir eventos infecciosos oportunistas en estos animales puede ser útil en la preservación de especies amenazadas. El objetivo de esta investigación fue aislar e identificar levaduras potencialmente patógenas del pelaje de osos hormigueros mantenidos en cautiverio. Se encuestó a 27 osos hormigueros, de la Fundación Parque Zoológico de São Paulo (FPZSP) y del Parque Municipal Quinzinho de Barros (Zôo-Sorocaba, SP). Catorce ejemplares serán oso hormiguero gigante (Mymercophaga tridactyla) y 13 oso hormiguero (Tamandua tetradactyla), de los cuales $63 \%$ machos y $37 \%$ hembras. Se utilizó la técnica de la alfombra cuadrada para obtener las muestras de piel. Las levaduras aisladas se identificaron por sus características morfológicas y por el método semiautomático ID-32 ${ }^{\circledR}$. Para describir las variables obtenidas a través del instrumento de investigación, se verificó la frecuencia de ocurrencia y los resultados se expresaron en valores relativos. Se aislaron un total de 33 levaduras de las muestras de piel de los 27 osos hormigueros. Las especies de levadura aisladas fueron: ocho Candida guilliermondii (24,2\%), tres C. famata $(9,1 \%)$, tres C. kefyr $(9,1 \%)$, dos C. glabrata $(6,1 \%)$, tres Cryptococcus laurentii $(9,1 \%)$, una C. humicola (3,0\%), seis Geotrichum candidum (18,2\%), três Malassezia pachydermatis $(9,1 \%)$, dos Rhodotorula glutinis $(6,1 \%)$ y dos Trichosporon asahii $(6,7 \%)$. Se puede concluir que las levaduras que se sabe que son patógenas pueden colonizar la microbiota del tegumento de osos hormigueros gigantes y osos hormigueros mantenidos en cautiverio y representan un riesgo potencial de infección oportunista para estos animales.

Palabras clave: Levaduras patógenas; Microbiota normal; Oso hormiguero gigante; Oso hormiguero bebé.

\section{Introdução}

O tamanduá-bandeira (Myrmecophaga tridactyla), um mamífero endêmico do cerrado brasileiro, é espécie atualmente considerada em vulnerabilidade de extinção. Entidades como a "União Internacional de Conservação da Natureza" (IUCN) incluem o tamanduá-bandeira em sua "Lista Vermelha” de espécies ameaçadas de extinção (IUCN, 2021). No Brasil, o tamanduá faz parte da "Lista Nacional das Espécies da Fauna Brasileira Ameaçadas de Extinção" (ICMBio, 2021) e é classificado como espécie "criticamente em perigo" pelo "Livro Vermelho da Fauna Ameaçada de Extinção no Estado do Paraná” (Braga, 2003). Pouco se conhece sobre a microbiota fúngica de espécimes selvagens, dessa forma, a oportunidade de se incluir esses animais neste trabalho caracteriza objeto de interesse científico. A realização da investigação sobre a presença de leveduras de potencial patogênico na microbiota de tamanduás, permitirá a implementação de futuros programas sanitários que tenham por objetivo a conservação daquelas e outras espécies ameaçadas de extinção (Bentubo et al, 2006).

A maior parte das evidências patológicas envolvendo tamanduás restringe-se a relatos de traumas, isto é, eventos de atropelamento em beira de estradas e casos de morte decorrente das consequências da destruição dos habitats (Weiss \& Vianna, 2012). Pouco se conhece acerca do status sanitário das espécies de tamanduá. Alguns autores têm se dedicado ao estudo de ectoparasitas nesses animais, como carrapatos, importantes vetores de doenças infecciosas para animais domésticos (Martins et al, 2004). Levantamentos realizados na região amazônica sugerem que os tamanduás também sejam portadores de Leishmania sp e Tripanossoma sp (Gottdenker et al, 2012; Muñoz-García et al, 2019). A presente pesquisa é dedicada ao estudo da presença e diversidade de leveduras de potencial patogênico, incluindo àquelas consideradas emergentes e oportunistas em quadros infecciosos na intenção de elucidar esse aspecto ecológico da colonização de tamanduás.

A microbiota fúngica que compõe a superfície corpórea dos seres vivos é constitutivamente dinâmica, ou seja, sofre periodicamente, mudanças qualitativas e/ou quantitativas. Essas mudanças decorrem, em grande parte, de fatores ambientais, como, localização geográfica, sanidade e condições climáticas (temperatura e o tempo de exposição à luz ultravioleta) (Araújo et al, 2003). Dentre os fungos que constituem a microbiota superficial do homem, as leveduras apresentam destacada 
importância, principalmente em indivíduos neutropênicos (Sun et al, 2015). Os fungos leveduriformes mais comumente isolados são: Candida spp. (Crocco et al, 2004; Oliveira et al, 2006), Rhodotorula spp. (Garcia-Martos et al, 2004; Lunardi et al, 2006; Riedel et al, 2007), Malassezia spp. (Scholottfeldt et al, 2002; Tarazooie et al, 2004; Oliveira et al, 2006) e Trichosporon spp. (Girmenia et al, 2005).

A emergência de novos patógenos fúngicos, mas de grande importância médica e veterinária, tem contribuído para o aumento da morbidade e mortalidade, especialmente em pacientes imunocomprometidos. Infecções fúngicas invasivas são causa significativa de morbidade e mortalidade em indivíduos imunocomprometidos (Fisher \& Sterbeck, 2005; Sun et al, 2015). Entre os pacientes humanos, aqueles com câncer, diabetes, transplantados de órgãos, etc, constituem uma nova população de doentes, dos fins do século XX e início do século XXI, que apresentam maior sobrevida devido a técnicas cirúrgicas mais agressivas, regimes terapêuticos com emprego de novos fármacos e diagnósticos mais aprimorados. Em Medicina Veterinária, a evolução dos protocolos terapêuticos para doenças alérgicas e a terapia anticâncer representam os principais eventos que permitiram aumentar a longevidade dos animais. Contudo, os fatores predisponentes às infecções fúngicas oportunistas ainda são pouco estudados e, por isso, pouco se conhece a seu respeito. Essa população da "nova era", ao mesmo tempo, que atinge maior longevidade, também está mais sujeita a infecções oportunísticas por patógenos emergentes (Giusiano et al, 2004; Silva \& Girmenia et al, 2005).

Uma vez que a microbiota residente/transitória caracteriza fonte de infecção para indivíduos imunocomprometidos, estudos ecológicos a respeito da diversidade de espécies de leveduras potencialmente patogênicas, como aquelas do gênero Trichosporon, que compõem a biota normal da superfície corpórea de animais representam importante passo no estudo da epidemiologia de infecções dessa natureza. O desconhecimento sobre a diversidade fenotípica e fatores associados à virulência das leveduras que ocorrem em nosso meio justificam a presente pesquisa. O objetivo da presente pesquisa foi investigar a frequência de isolamento de leveduras potencialmente patogênicas e consideradas emergentes em infecções oportunistas que compõem a microbiota superficial do pelame de tamanduás mantidos em cativeiro, bem como, caracterizar os isolados leveduriformes por meio de kit comercial semi-automatizado ID $32 \mathrm{C}^{\circledR}\left(\right.$ Biomerieux $\left.^{\circledR}\right)$.

\section{Metodologia}

\subsection{Animais}

Leveduras patogênicas oportunistas foram pesquisadas em 27 tamanduás, provenientes da Fundação Parque Zoológico de São Paulo (FPZSP) e Parque Municipal Quinzinho de Barros (Zôo-Sorocaba, SP), ambos, integrantes do projeto de pesquisa multicentralizado idealizado pelo Grupo de Trabalho pela Conservação do Tamanduá no Brasil (GCTB-IBAMA). Catorze espécimes de tamanduá-bandeira (Mymercophaga tridactyla) e 13 espécimes de tamanduá-mirim (Tamandua tetradactyla), dos quais, $63 \%$ eram machos e 37\%, fêmeas serão incluídos na presente investigação. A contenção dos animais será realizada por técnicos designados pela coordenação geral do projeto e seguirá em acordo com os protocolos estabelecidos pelos médicos veterinários responsáveis pelos animais em suas respectivas instituições de albergue.

\subsection{Colheita das amostras}

As amostras foram obtidas por meio da fricção de quadrados de carpete (Mariat \& Adam-Campos, 1967; Bentubo et al, 2006) no pelame do dorso dos animais estudados. Quadrados de carpete de, aproximadamente, $5 \times 5$ centímetros foram lavados com água e detergente neutro, enxaguados e mantidos em água corrente por 24 horas para garantir que estivessem livres de quaisquer resíduos de produtos químicos. Em seguida, os carpetes foram envolvidos em um invólucro composto por duas camadas. A camada interna era de papel alumínio e a externa de papel pardo. Cada quadrado de carpete foi esterilizado 
em autoclave para uso. A caracterização das amostras foi realizada no Laboratório de Biologia Molecular e Celular (LBMC) da Universidade Paulista -UNIP (CLININFEC-CNPq), São Paulo, SP, Brasil.

\subsection{Caracterização morfológica dos isolados}

Todas as amostras foram semeadas uniformemente em placas de Petri contendo ágar Sabouraud dextrose (ASD) $\left(\right.$ Difco $\left.^{\circledR}\right)$ acrescidas de $0,5 \%$ de cloranfenicol e incubadas a temperatura ambiente até que fosse evidenciado crescimento microbiano. Cada colônia foi agrupada de acordo com sua semelhança macroscópica, isolada e repicada em triplicata para tubos contendo o mesmo meio; repiques periódicos eram realizados para manter a viabilidade das amostras. Todos os cultivos leveduriformes foram submetidos à técnica de microcultivo em lâmina conforme preconiza Porto et al (1981). Cada amostra foi semeada em estrias sobre lâminas contendo meio de ágar-fubá acrescido de Tween 80. Posteriormente, as estrias foram cobertas com lamínula de vidro e cada placa será incubada a $30^{\circ} \mathrm{C}$ durante 24 horas e submetida à leitura para verificação de suas características morfológicas microscópicas (Kurtzman \& Fell, 1998; De Hoog et al, 2004).

\subsection{Caracterização bioquímica dos isolados}

Todas as amostras foram testadas para a produção de urease em meio de ágar uréia de Christensen (Cristensen, 1946) e para a fermentação de carboidratos (Wickerham, 1951). Cepas-padrão de Cryptococcus neoformans (ICB 162C) e Candida albicans (ICB 12A) foram utilizadas como controles positivo e negativo, respectivamente.

\subsection{Identificação semi-automatizada das leveduras (ID 32 C, Biomerieux ${ }^{\circledR}$ )}

Para a realização das provas de identificação de leveduras pelo método ID $32 \mathrm{C}$ (Biomerieux ${ }^{\circledR}$ ), os isolados foram semeados em ASD e incubados por $24-72 \mathrm{~h}$ a $30^{\circ} \mathrm{C}$. Suspensão de microrganismos será preparada em tubos contendo $2 \mathrm{~mL}$ de água destilada esterilizada de acordo com o tubo 2,0 da escala de MacFarland. Duzentos e cinqüenta microlitros (250 $\mu \mathrm{L})$ de cada inóculo serão dispensados numa ampola contendo meio de assimilação de carbono. Após homogeneização, 135 $\mu \mathrm{L}$ de cada diluição serão adicionados a cada poço da galeria de ID $32 \mathrm{C}$, as quais foram incubadas em câmara úmida sob $30{ }^{\circ} \mathrm{C}$ de temperatura, durante $72 \mathrm{~h}$ e submetidas à leitura a cada $24 \mathrm{~h}$, conforme orientação do fabricante. Foram consideradas positivas, as amostras que apresentarem turvação superior a do controle negativo. O perfil numérico obtido na leitura visual será analisado pelo programa Apiweb ${ }^{\circledR}$ (Fricker-Hidalgo et al, 1996). Foram considerados identificados os isolados que apresentaram níveis de acurácia $\geq 85 \%$ à leitura no programa.

\subsection{Análise dos resultados}

Para descrever as variáveis obtidas por meio do instrumento de pesquisa, foi verificada a frequência de ocorrência de cada espécie de levedura dentro do total de isolados obtidos. Os resultados foram expressos em números absolutos (N) e relativos $(\%)$.

\section{Resultados e Discussão}

Em relação às espécies silvestres, ainda são pouco expressivos os trabalhos de pesquisa relacionados aos aspectos elementares da sanidade desses animais. A crescente preocupação com o meio ambiente e a necessidade da conservação da fauna brasileira endêmica tem estimulado iniciativas pioneiras por todo o país. É o caso do Grupo de Trabalho pela Conservação do Tamanduá no Brasil (GCTB) que, sediado na Fundação Parque Zoológico de São Paulo (FPZ-SP), tem como missão promover ações que favoreçam a conservação das espécies de tamanduá no Brasil. Por meio de uma colaboração científica estabelecia entre GCTB e o Laboratório de Biologia Molecular e Celular (LBMC) da Universidade Paulista -UNIP 
onde também está sediado o CLININFEC (Grupo de Pesquisa em Clínica e Doenças Infecciosas, cadastrado no CNPq) foi desenvolvido o presente trabalho.

Foram isoladas 33 leveduras a partir das amostras de pelame dos 27 tamanduás investigados. Mais de um isolado leveduriforme por amostra foi obtido em alguns casos, como pode ser observado na Tabela 1. As demais espécies isoladas foram: Candida guilliermondii $(\mathrm{N}=8)$, C. famata $(\mathrm{N}=3), C$. kefyr $(\mathrm{N}=3), C$. glabrata $(\mathrm{N}=2)$, Cryptococcus laurentii $(\mathrm{N}=3), C$. humicola $(\mathrm{N}=1)$, Malassezia pachydermatis $(\mathrm{N}=3)$, Geotrichum candidum $(\mathrm{N}=6)$, Rhodotorula glutinis $(\mathrm{N}=2)$ e Trichosporon asahii $(\mathrm{N}=2)$.

Tabela 1: Isolamento de leveduras da microbiota do pelame de 27 tamanduás sadios provenientes da Fundação Parque Zoológico de São Paulo (São Paulo, SP, Brasil) e Parque Municipal Quinzinho de Barros (Sorocaba-SP, Brasil).

\begin{tabular}{|c|c|c|c|c|}
\hline Espécie & Sexo & $\mathrm{N}^{\circ}$ amostra & Identificação da amostra & Identificação do isolado \\
\hline T. bandeira & Macho & 01 & 28666 & Rhodotorula glutinis \\
\hline T. bandeira & Fêmea & 02 & 14777 & Candida kefyr \\
\hline T. bandeira & Macho & 03 & 22760 & Candida famata, Trichosporon asahii \\
\hline T. bandeira & Macho & 04 & 25106 & Candida guilliermondii \\
\hline T. bandeira & Macho & 05 & 16446 & Geotrichum candidum \\
\hline T. bandeira & Macho & 06 & 22759 & Cryptococcus laurentii \\
\hline T. bandeira & Macho & 07 & 27109 & Cryptococcus laurentii \\
\hline T. mirim & Macho & 08 & 25848 & Cryptococcus humicola, Malassezia pachydermatis \\
\hline T. mirim & Macho & 09 & 28097 & Candida kefyr \\
\hline T. mirim & Macho & 10 & 20979 & Candida famata \\
\hline T. mirim & Fêmea & 11 & 20679 & Candida guilliermondii \\
\hline T. mirim & Macho & 12 & 28948 & Candida glabrata \\
\hline T. mirim & Fêmea & 13 & 28949 & Geotrichum candidum \\
\hline T. bandeira & Macho & 14 & MC38484 & Candida guilliermondii \\
\hline T. mirim & Fêmea & 15 & 22442 & Candida guilliermondii \\
\hline T. mirim & Macho & 16 & 23885 & Geotrichun candidum, Malassezia pachydermatis \\
\hline T. mirim & Macho & 17 & 28639 & Candida guilliermondii, Trichosporon asahii \\
\hline T. mirim & Fêmea & 18 & 28869 & Candida guilliermondii \\
\hline T. bandeira & Macho & 19 & TB001 & Candida glabrata \\
\hline T. bandeira & Macho & 20 & TB002 & Candida kefyr \\
\hline T. bandeira & Fêmea & 21 & TB003 & Candida famata \\
\hline T. bandeira & Fêmea & 22 & TB004 & Geotrichun candidum \\
\hline T. bandeira & Fêmea & 23 & TB005 & Geotrichum candidum \\
\hline T. bandeira & Fêmea & 24 & TB006 & Candida guilliermondii \\
\hline T. mirim & Fêmea & 25 & TM001 & Candida guilliermondii, Malassezia pahydermatis \\
\hline T. mirim & Macho & 26 & TM002 & Cryptococcus laurentii, Rhodotorula glutinis \\
\hline T. mirim & Macho & 27 & TM003 & Geotrichun candidum \\
\hline
\end{tabular}

Fonte: Laboratório de Biologia Molecular e Celular/CLININFEC-CNPq (UNIP), São Paulo, SP, Brasil.

A técnica do quadrado de carpete friccionado sobre o dorso, originalmente descrita por Mariat \& Adam-Campos (1967) para a obtenção de amostras para o isolamento de fungos filamentosos. Seu emprego para essa finalidade já foi testado e comprovado em trabalhos realizados anteriormente (Bentubo et al, 2006). A técnica clássica também se mostrou bastante eficiente para a obtenção de isolados leveduriformes, tal qual foi possível observar na presente investigação. Por meio da análise da morfologia macroscópica dos isolados obtidos em cultura foi possível estabelecer correspondência com o provável gênero da levedura. Para a confirmação das suspeitas foram realizados testes adicionais de cultivo em lâmina, para a avaliação da morfologia microscópica e método semi-automatizado por kit ID-32 ${ }^{\circledR}$ (Biomerieux ${ }^{\circledR}$ ), para averiguação das características fisiológicas dos isolados. Esses métodos, quando utilizados em associação, permitem maior acurácia na identificação específica de fungos leveduriformes (Lacaz et al, 2002).

Dos cinco isolados que apresentaram coloração branca, textura lisa e aspecto úmido e opaco que, inicialmente, se mostraram compatíveis com leveduras do gênero Candida sp, apenas três puderam ser identificados como pertencentes a gênero suspeito: um de Candida famata, um de Candida guilliermondii e um de Candida kefyr. Um dos isolados apresentou estrutura de natureza hidrofóbica observada na superfície das células no teste de tinta nankin e foi identificado pelo kit ID$32 \mathrm{C}^{\circledast}\left(\right.$ Biomerieux $\left.^{\circledR}\right)$ como Cryptococcus laurentii. E por último o isolado caracterizado pela textura mais membranosa, assim 
como se suspeitava, foi identificado como um Geotrichum candidum, pelo mesmo método (Lacaz et al, 2002). Nesta pesquisa, outras leveduras, como Candida spp., Geotrichum sp, Cryptococcus spp. e Rhodotorula sp, também foram isoladas do pelame dos animais submetidos à investigação. Dessas leveduras, os gêneros Candida e Cryptococcus, merecem destaque especial, por constituírem patógenos importantes.

As leveduras do gênero Candida (C. famata, C. glabrata, C. guilliermindii e C. kefyr) foram isoladas da maior parte da população de tamanduás pesquisada $(59,2 \%)$, sugerindo que estas sejam as leveduras mais comumente encontradas colonizando a superfície do corpo de tamanduás. Embora $C$. albicans seja a espécie mais relatada em casos de infecção envolvendo animais, as novas condições de vida capazes de produzir estresse e, consequentemente, imunossupressão promovem a emergência de novas espécies em processos patológicos, os quais serão gradativamente referenciados na literatura médico-veterinária (Greene, 2006). Cryptococcus spp. são patógenos reconhecidos em medicina humana. Esse gênero apresentou frequência de isolamento de 13, 3\% entre os tamanduás. A literatura sugere que pombos e outras aves sejam importantes disseminadoras de C. neoformans, espécie relevante em doença humana. Se levarmos em conta que a maior parte dos parques zoológicos do mundo sejam, frequentemente, visitados por aves migratórias e que espécies de Cryptococcus, tais como C. laurentii, verificadas neste trabalho, já tenham sido isoladas a partir de fezes de aves migratórias como o ganso canadense (Branta canadensis), talvez não seria improvável a obtenção de isolados da microbiota transitória superficial de animais residentes daqueles recintos visitados (Filion et al, 2006; Rosário et al, 2008). A imunossupressão constitui fator determinante para a ocorrência de infecções oportunistas. Como já foi dito, enfermidades de base com característica imunocomprometedora (Aids, câncer, diabetes, transplante de órgãos, antibióticoterapia e/ou corticoterapia prolongadas) são fatores predisponentes bastante conhecidos, especialmente para seres humanos. Além disso, a caça predatória, tráfico e o cativeiro são situações potencialmente imunossupressoras, dado o nível de estresse às quais os animais envolvidos estão sujeitos (Tashiro et al, 1995; Kremery et al, 1999).

O gênero Malassezia sp compreende diversas espécies, muitas delas responsáveis pelas otites e dermatites em cães e gatos. Porém, existem poucos registros da presença delas como colonizadoras da microbiota de animais selvagens, e nenhum especificamente relacionado a tamanduás. O presente trabalho obteve como resultado o isolamento de Malassezia pachydermatis em três tamanduás-mirim (11,1\%). Em 2006, Coutinho et al avaliaram amostras de cerume de 132 felídeos selvagens adultos e saudáveis mantidos em cativeiro. O grupo foi dividido em grandes e pequenos felídeos, os quais tinham uma amostragem de 55 e 77 animais, respectivamente. O primeiro grupo teve resultados positivos somente para Malassezia sympodialis em 56,9\% dos indivíduos, enquanto o segundo grupo mostrou positividade exclusivamente para Malassezia pachydermatis em 43,1\% deles. Dentre o grupo de grandes felídeos, os leões foram a espécie que apresentou maior incidência de leveduras. De 26 animais, 25 (96,2\%) foram positivos para M. sympodialis. Gandra et al (2008) isolaram Malassezia spp. do meato acústico de 24 (80\%) morcegos insetívoros (Molossus molossus) da região de Montenegro, em Rondônia, sendo a espécie mais frequentemente encontrada, Malassezia pachydermatis, isolada em 15 animais (62,5\%), seguida pela M. furfur, em cinco (20,8\%), M. globosa, em três (12,5\%) e M. sympodialis, em um indivíduo (4,2\%). Em Pisa, na Itália, existem estudos que relatam a presença de espécies de Malassezia sp no conduto auditivo externo de suídeos saudáveis pertencentes a diferentes raças e idades (oito meses à quatro anos). Para este estudo foram utilizados 185 javalis selvagens, 107 animais de raças de grande porte e 116 da raça Cinta Senese. Os resultados demonstraram que Malassezia pachydermatis foi a única espécie isolada na raça Cinta Senese (20,7\%), em indivíduos jovens de raças de grande porte (13,6\%) e em javalis (12,9\%). Nos animais adultos de grande porte foi possível o isolamento e caracterização das espécies $M$. sympodialis (63,6\%) e $M$. furfur (22,7\%) (Nardoni et al, 2010). Até o momento, os estudos apresentados possuem algumas características relevantes em comum. 
O presente trabalho, assim como os de Coutinho et al (2006) e Nardoni et al (2010), utilizaram como amostragem, em sua grande maioria, animais provenientes de cativeiro. Gandra et al (2008) teve como base de seu estudo animais de vida livre, que foram capturados para a realização do mesmo. Contudo, em nenhum dos dois casos os indivíduos utilizados apresentavam sinais clínicos de infecção causada pelo fungo. Esse aspecto também foi observado em nossos animais. Outro ponto importante a ser discutido é a frequência de isolamento de espécies da levedura em questão. Por meio da análise dos dados obtidos observou-se a prevalência de Malassezia sympodialis nos resultados de Coutinho et al (2006) e Nardoni et al (2010). Já no presente estudo, assim como nos demais trabalhos apresentados, Malassezia pachydermatis foi a única espécie isolada do pelame, indicando ser esta predominante nesse microambiente. Outra questão relevante seria a metodologia de crescimento em meio de cultura utilizada, que pode não ter sido a ideal para o crescimento de outras espécies de Malassezia sp.

Em consonância às suspeitas levantadas, foi possível confirmar que o isolado que apresentou coloração branca, textura rugosa e aspecto seco e opaco compatíveis com o de uma levedura do gênero Trichosporon sp. era, de fato, um $T$. asahii (Guého et al, 1994; Kurtzman \& Fell, 1998; De Hoog et al, 2004). Assim como aquele isolado, o mais distinto de todos, que exibia coloração alaranjada, textura lisa e aspecto úmido e brilhante, compatível com o gênero Rhodotorula foi identificado como R. glutinis. Vale ressaltar que também foi possível observar a presença discreta de uma substância de natureza hidrofóbica na superfície das células de Rhodotorula glutinis no teste de tinta nanquim (Kurtzman \& Fell, 1998). Segundo autores renomados, a presença de cápsula pode ser observada não apenas em leveduras do gênero Cryptococcus como também em Rhodotorula spp. (De Hoog et al, 2004).

Segundo a literatura, Trichosporon spp. já foram isoladas do solo e da água em ambiente selvagem (Paula et al, 1983; Martins et al, 1989), o que justifica os isolamentos obtidos nesse trabalho. No entanto, levando-se em conta que os animais estudados vivem em condição de cativeiro, ou seja, são rotineiramente expostos ao contato com tratadores, médicos veterinários e outros profissionais da área, que também podem albergar essa levedura na sua microbiota, não se pode ignorar a possibilidade desses indivíduos exercerem papel como agentes disseminadores de agentes microbianos diversos, entre eles, $T$. asahii, espécie que além de ser a mais importante nos casos de infecção humana oportunística (Fleming et al., 2002; Madariaga et al, 2003), também é considerada agente comum do ambiente (Sugita et al, 2000). A literatura menciona apenas uma descrição de Trichosporon sp como agente patogênico de piedra branca superficial em primatas não humanos; entretanto, acredita-se que a ocorrência desses processos superficiais nos animais seja subestimada em virtude, principalmente, da carência de relatos na literatura (Kaplan, 1959). Ainda não existem descrições de infecção causada por T. asahii em animais domésticos ou silvestres, de qualquer espécie. No entanto, a presença do microrganismo na microbiota associada à possível condição de imunossupressão, expõe os animais aos mesmos riscos aos quais estão expostos os seres humanos.

A macromorfologia das colônias não pode ser considerada como critério único para a identificação dos isolados obtidos a partir das amostras colhidas do pelame dos tamanduás. Nossas observações foram compatíveis com as de vários autores da escola clássica de micologia (Lacaz et al, 2002), confirmando que é imprescindível recorrer a outras características como morfologia microscópica e comportamento fisiológico dos isolados [o kit de identificação ID-32C ${ }^{\circledR}$ (Biomerieux ${ }^{\circledR}$ ) deverá auxiliar de maneira relevante nesse processo de identificação], bem como aos demais testes complementares disponíveis na composição de um repertório viável, mais criterioso e consistente para a identificação taxonômica de microrganismos, sejam eles provenientes de processos infecciosos ou simplesmente membros da microbiota residente/transitória de um espécime. Também cabe comentar que foi observado o isolamento de outros gêneros fúngicos, com característica filamentosa, considerados nessa pesquisa como contaminantes devido ao seu caráter sapróbio. É importante ressaltar que os animais utilizados neste estudo não mostraram nenhum tipo de alteração clínica, assim como ocorre com aqueles citados em outros estudos de referência. 


\title{
4. Considerações Finais
}

Em relação à origem dos animais utilizados, sabe-se que eram provenientes da Fundação Parque Zoológico de São Paulo e do Zoológico Municipal Quinzinho de Barros, em Sorocaba. Visto que os animais estão fora de seu ambiente natural e que o estresse pode influenciar na colonização dos animais, trabalhos com espécimes de vida livre devem ser estimulados a fim de que seja possível estabelecer parâmetros mais contundentes para as espécies estudadas considerando, inclusive, outros tipos de microbiota potencialmente patogênicas, como por exemplo, aquela constituída por fungos filamentosos e bactérias de importância médico-veterinária. Tamanduás mantidos em cativeiro são portadores de leveduras com importante potencial patogênico, assim sendo, para manter o sucesso de programas de preservação das espécies, esses animais devem também ter sua microbiota monitorada devido aos fatores predisponentes que podem incidir sobre o meio onde eles estão inseridos e, quanto ao surgimento de manifestações clínicas de infecções oportunistas. O emprego de técnicas semi-automatizadas, quando empregadas em associação com técnicas clássicas produzem resultados de identificação muito satisfatórios. Malassezia pachydermatis também é levedura integrante da microbiota residente e/ou transitória normal do pelame de tamanduás-mirim à semelhança do que se observa em estudos realizados com outros mamíferos. Outro ponto de relevância seria a não observância de outras espécies desse gênero de levedura, o que na opinião dos autores requer mais investigações, já que, novas espécies têm sido cada vez mais comumente identificadas na microbiota de animais de diferentes espécies. Talvez a adoção de meios de cultura mais adequados para a rotina de isolamento de espécies lipodependentes possa contribuir para elucidação desse caráter ecológico de colonização por Malassezia spp. no pelame de tamanduás.

\section{Agradecimentos}

\author{
À Pró-Reitoria de Pesquisa e Pós-Graduação da Universidade Paulista - UNIP, São Paulo, SP, Brasil.
}

\section{Referências}

Araújo, A. J. G., Bastos, O. M. P., Souza, M. A. J. \& Oliveira, J. C. (2003). Ocorrência de onicomicose em pacientes atendidos em consultórios dermatológicos da cidade do Rio de Janeiro, Brasil. An Bras Dermatol, 78, 445-455.

Bentubo, H. D. L., Fedullo, J. D. L., Corrêa, S. H. R., Teixeira, R. H. F. \& Coutinho, S. D. (2006). Isolation of Microsporum gypseum from the haircoat of helth wild felids kept in captivity in Brazil. Braz J Microbiol, 37, 148- 152.

Braga, F. G. (2003). Tamanduá-bandeira (Myrmecophaga tridactyla), espécie em perigo: uma preocupação no Estado do Paraná. Acta Biol, 33 , $193-194$.

Christensen, W. B. (1946). Urea decomposition as a means of differentiating Proteus and Paracolon cultures from Salmonella and Shigella types. J Bacteriol, $52,461-466$.

Coutinho, S. D., Fedullo, J. D. \& Corrêa, S. H. (2006). Isolation of Malassezia spp. from cerumen of wild felids. Sabouraudia, 44(4), $383-387$.

Crocco, E. I., Mimica, L. M. J.; Muramatu, L. H., Garcia, C., Souza, V. M., Ruiz, L. R. B. \& Zaitz, C. (2004). Identificação de espécies de Candida e susceptibilidade antifúngica in vitro: estudo de 100 pacientes com candidíases superficiais. An Bras Dermatol, 79, 689-697.

De Hoog, G. S., Guarro, J., Gené J. \& Figueiras, M. J. (2004). Atlas of Clin.Fungi. (No. Ed. 2), Ultrech \& Réus: Centralbureal voor Schimmelcultures to IUniversitat Rovira, Virgilli.

Filion, T., Kidd, S. \& Aguirre, K. (2006). Isolation of Cryptococcus laurentii from Canada Goose guano in rural upstate New York. Mycopathologia, 162, 363-368.

Fischer, L. \& Sterneck, M. (2005). Invasive fungal infections in patients after liver transplantation. Mycoses, 48, 27-35.

Fleming, R. V., Walsh, T. J. \& Anaissie, E. J. (2002). Emerging and less common fungal pathogens. Infect Dis Clin North Am, 16, $915-933$.

Fricker-Hidalgo, H., Vandapel, O., Duchesne, M. A., Mazoyer, M. A., Monget, D., Lardy, B., Ledeau, B., Freney, J., Ambroise-Thomas, P. \& Guillot, R. (1996). Comparision of the new API Candida system to the ID 32 C system for identification of clinical important species. J Clin Microbiol, $34,1846-1848$.

Gandra, R. F., Simão, R. C. G., Matsumoto, E. F., Silva, B. C. M., Ruiz, L. S., Silva, E. G., Gambale, W. \& Paula, C. R. (2006). Genotyping by RAPD-PCR analyses of Malassezia furfur strains from pityriasis versicolor and seborrhoeic dermatitis patients. Mycopathologia, 162, 273-280.

García-Martos, P., García-Agudo, L., Ruiz-Aragón, J., Saldarreaga, A. \& Marín, P. (2004). Asimilación de carboidratos por cepas de Rhodotorula glutinis de origem clínica y ambiental. Rev Iberoam Micol, 21, 90-92. 
Girmenia, C., Pagano, L., Martino, B., D’antonio, D., Fanci, R., Specchia, G., Melillo, L., Buelli, M., Pizzarelli, G., Venditti, M. \& Martino, P. (2005). Invasive infections caused by Trichosporon species and Geotrichum capitatum in patients with hematological malignancies a retrospective multicenter study from Italy and review of the literature. J Clin Microbiol, 43, 1818-1828.

Giusiano G. E., Mangiaterra M., Rojas, F. \& Gómes, V. (2004). Yeasts species distribution in neonatal intensive care units in northeast Argentina. Mycoses, 47, 300-303.

Gottdenker, N. L., Chaves, L. F., Calzada, J. E., Saldaña, A. \& Carroll, C. R. (2012). Host life history strategy, species diversity, and habitat influence Trypanosoma cruzi vector infection in changing landscapes. PLoS neglected trop dis, 6(11), e1884.

Greene, C. E. (2006). Infectious diseases of the dog and cat (No. Ed. 3). WB SaunderslElsevier Science.

Guého, E., Improvisi, L., De Hoog, G. S. \& Dupont, B. (1994). Trichosporon on humans: a pratical account. Mycoses, 37, 3-10.

Instituto Chico Mendes De Conservação Da Biodiversidade. (2021). Lista de espécies ameaçadas de extinção. https://www.icmbio.gov.br/portal/faunabrasileira/2741-lista-de-especies-ameacadas-saiba-mais.html

International Union For Conservation Of Nature. (2021). The IUCN red list of threatened species. www.https://www.iucnredlist.org/

Kaplan, W. (1959). Piedra in lower animals; a case report of white piedra in a monkey and a review of literature. J Am Vet Med Assoc, 134, $113-117$.

Kremery, V., Mateicka, F. Jr., Kunova, A., Spanik, S., Gyarfas, J., Sycova, Z. \& Trupi, J. (1999). Hematogenous trichosporonosis in cancer patients: report of 12 cases including five during prophylaxis with itraconazole. Supp Care Cancer, 7, 39-43.

Kurtzman, C., Fell, J. W. \& Boekhout, T. (Eds.). (2011). The yeasts: a taxonomic study. Elsevier.

Lacaz, C. D. S., Porto, E., Martins, J. E. C., Heins-Vaccari, E. M., \& Takahashi de Melo, N. (2002). Tratado de micologia médica.

Lunardi, L. W., Aquino, V. R., Zimerman, R. A. \& Goldani, L. Z. (2006). Epidemiology and outcome of Rhodotorula fungemia in a tertiary care hospital. Clin Infect Dis, 43, 60-63.

Madariaga, M. G., Tenorio, A. \& Proia, L. (2003). Trichosporon inkin peritonitis treated with caspofungin. J Clin Microbiol, 41, 5827-5829.

Mariat, F. \& Adam-Campos, C. (1967). La technique du carré du tapis, méthode simple de prélevement dans les mycoses superficielles. Ann Inst Pasteur, 113, $666-668$.

Martins, M. T., Gambale, W., Paula, C. R. \& Pellizari, V. H. (1989). Utilização de bactérias e fungos como indicadores na avaliação de fatores fisiográficos que interferem nos processos de autodepuração de um córrego subtropical. Rev Microbiol, 20, 278-291.

Martins, J.R., Medri, I. M., Oliveira, C. M. \& Guglielmone, A. (2004). Ocorrência de carrapatos em tamanduá-bandeira (Myrmecophaga tridactyla) e tamanduá- mirim (Tamandua tetradactyla) na região do Pantanal Sul Mato-Grossense, Brasil. Ciênc Rural, 34, 293-295.

Muñoz-García, C. I., Sánches-Montes, S., Villanueva-García, C., Romero-Callejas, E., Díaz-Lopez, H. M., Gordillo-Chávez, E. J., Martínez-Carrasco, C., Berriatua, E. \& Rendón-Franco, E. (2019). The role of sloths and anteaters as Leishmania spp. reservoirs: a review and a newly described natural infection of Leishmania mexicana in the northern anteater. Parasitol Res, 118(4), 1095-1101.

Nardoni, S., Merildi, V., Frangioni, S., Ariti, G., Verin, R., Vannucci, P. \& Mancianti, F. (2010). Isolation and characterization of Malassezia spp. in healthy swine of different breeds. Vet Microbiol, 141(1-2), 155-158.

Oliveira, J. A., Barros, J. A., Cortez, A. C. A. \& Oliveira, J. S. R. J. (2006). Micoses superficiais na cidade de Manaus, AM, entre março e novembro/2003. An Bras Dermatol, 81, 238-243.

Paula, C. R., Purchio, A. \& Gambale, W. (1983). Yeasts from beaches in the Southern area of São Paulo, Baixada Santista, Brazil. Rev Microbiol, 14, 136-143.

Porto, E., Takahashi, N., Heins, E. M. \& Lacaz, C. da S. (1981). Mieno método para microcultivo de hongos. Rev Argent Micol, 4, 24-29.

Riedel, D. J., Johnson, J. K. \& Forrest, G. N. (2007). Rhodotorula glutinis fungemia in a liver-kidney transplant patient. Transpl Infect Dis, 10, 197-200.

Rosario, I., Acosta, B. \& Colom, F. (2008). La paloma y otras aves como reservorio de Cryptococcus spp. Rev Iberoam Micol, 25 , S13-S18.

Schlottfeldt, F. S., Tramontin, S. W., Nappi, B. P. \& Santos, J. I. (2002). Reclassificação taxomômica de espécies do gênero Malassezia: revisão da literatura sobre as implicações clinicolaboratoriais. J Bras Patol Méd Lab, 38, 199-204.

Sugita, T., Nishikawa, A., Ichikawa, T., Ikeda, R. \& Shinoda, T. (2000). Isolation of Trichosporon asahii from environmental materials. Med Mycol, 38, 2730 .

Sun, Y., Huang, H., Chen, J., Li, J., Ma, J., Li, J., Yu, K., Hu, J., Jin, J., Wang, C., Wu, D., Xiao, Y. \& Huang, X. (2015). Invasive fungal infection in patients receiving chemotherapy for hematological malignancy: a multicenter, prospective, observational study in China. Tumor Biol, 36(2), 757-767.

Tashiro, T., Nagai, H., Nagaoka, H., Goto, Y., Kamberi, P. \& Nasu, M. (1995). Trichosporon beigelii pneumonia in patients with hematologic malignancies. Chest, 108, 190-195.

Tarazooie, B., Kordbacheh, P., Zaini, F., Zomorodian, K., Saadat, F., Zeraati, H., Hallaji, Z. \& Rezaie, S. (2004). Study of the distribution of Malassezia species in patients with pityriasis versicolor and healthy individuals in Tehran, Iran. BMC Dermatol, 4, 1-6.

Weiss, L. P. \& Vianna, V. O. (2012). Levantamento do impacto das rodovias BR-376, BR-373 e BR-277, trecho de Apucarana a Curitiba, Paraná, no atropelamento de animais silvestres. Publ. UEPG Ci. Biol Saúde, 18, 121-133.

Wickerham, L. J. (1951). Taxonomy of yeasts (No. 1029). US department of agriculture. 